\title{
A Qualitative Analysis of Problematic and Non-problematic Alcohol Use After Bariatric Surgery
}

\author{
Danielle L. Reaves $^{1}$ (D) Joanne M. Dickson ${ }^{1,2} \cdot$ Jason C. G. Halford $^{1} \cdot$ Paul Christiansen $^{1} \cdot$ Charlotte A. Hardman $^{1}$
}

Published online: 3 April 2019

(C) The Author(s) 2019

\begin{abstract}
Objectives Bariatric surgery is an effective weight loss tool, but an under-communicated side effect may include the increased risk for alcohol problems. Few studies have examined contributors towards alcohol problems following surgery using a qualitative approach. Therefore, the current study aimed to generate insight informed by participants with problematic alcohol use following bariatric surgery, in comparison with participants without.

Methods Participants (14; females, $n=9$; males, $n=5$ ) completed semi-structured interviews using questions relating to alcohol use, relationship to food, support and surgical experiences. Thematic analysis was conducted to provide insight into the factors which influenced drinking behaviours that participants engaged in following bariatric surgery, and motivations for drinking or limiting alcohol.

Results Five core themes were identified between both participants with and without problematic alcohol use: (1) drinking motivations, (2) self-image, (3) impact of restriction on eating behaviour, (4) support needs and (5) surgical preparedness. A sixth core theme ("resilience") was identified specifically amongst participants without problematic alcohol use. Divergent experiences, cognitions and behaviours formed sub-themes within the five core themes and highlighted the differences between participants with and without problematic alcohol use within the core themes.

Conclusion This study is the first to qualitatively assess themes relating to the development of problematic alcohol use after bariatric surgery while additionally using a comparison group without problematic alcohol use. The findings highlight key features which contribute to problematic alcohol use, as well as experiences and cognitions that may be helpful in preventing this phenomenon in bariatric populations.
\end{abstract}

Keywords Qualitative $\cdot$ Bariatric surgery $\cdot$ Drinking to cope $\cdot$ Resilience $\cdot$ Alcohol use

Danielle L. Reaves

Dreaves@liverpool.ac.uk

Joanne M. Dickson

j.dickson@ecu.edu.au

Jason C. G. Halford

J.C.G.Halford@liverpool.ac.uk

Paul Christiansen

Paul.Christiansen@liverpool.ac.uk

Charlotte A. Hardman

Charlotte.Hardman@liverpool.ac.uk

1 Department of Psychological Sciences, University of Liverpool, Eleanor Rathbone Building, Bedford Street South, Liverpool L69 7ZA, UK

2 Psychology Department, Edith Cowan University, Perth 6027, Australia

\section{Introduction}

Bariatric surgery is offered as an effective intervention to reduce severe obesity wherein patients are expected to lose an average of $60 \%$ of their excess weight, varying by procedure $[1,2]$. Although bariatric surgery represents a tool for weight reduction, the intervention is biologically based. Therefore, potential underlying psychological contributors to excessive weight could be left unaddressed, giving rise to further complications [3]. Recent studies have raised concerns than an under-communicated side effect of bariatric surgery may include increased risk for alcohol misuse, especially following Roux-en-Y gastric bypass (RYGB) [4-6]. This is possibly attributable to physiological changes in alcohol absorption, which produce increased sensitivity to the effects of alcohol [7]. However, physiological changes are unlikely to increase alcohol use in isolation, as they are experienced by most 
individuals while only a (sizable) minority develop patterns of alcohol misuse. Instead, evidence from several large, longterm cohort studies points to individual differences in increasing risk for post-surgery alcohol misuse. Specifically, male gender, smoking, regular alcohol use before surgery, younger age, recreational drug use and lower sense of belonging all increase the risk for post-surgical alcohol misuse $[5,6]$. However, this evidence is mostly reliant on demographic measures, and the psychological mechanisms in post-surgical alcohol misuse remain under-explored.

Identifying contributors to alcohol-related outcomes following surgery is critical to understanding the psychological motivators of alcohol misuse. In general, bariatric candidates with known/suspected psychiatric illness, substance misuse or dependence are advised to eliminate alcohol after surgery to reduce the risk of alcohol misuse [8]. While there remains a paucity of research examining to what extent patients follow this advice, alcohol misuse tends to manifest around 24 months post-surgery and persists further onwards [4-6]. Importantly, there are "new onset" instances, where alcohol misuse is not observed until after surgery [9], while other patients with "high risk" drinking before surgery subsequently discontinue $[10$, 11]. Differing alcohol use may be the product of underlying motivational processes that increase alcohol use when surgeryinduced restriction disrupts usual eating patterns. Coping is one of several identified motivations for eating foods with a high hedonic value (i.e. high-fat, sugar or calorie-dense foods). Eating to cope represents a behavioural response to mitigate negative states or circumstances, such as to forget about worries (i.e. negative reinforcement) [12]. Interestingly, eating to cope is associated with a higher body mass index (BMI), even while controlling for similar constructs like addictive-like eating or binge eating $[12,13]$. Critically, motivations to drink alcohol share characteristics with those driving hedonic eating [12], and drinking to cope predicts alcohol consumption after a stressor when an individual has fewer adaptive coping strategies [14]. Further, increasing alcohol use through drinking to cope has been observed in populations with specific personality traits or qualities, including higher anxiety, hopelessness and depression $[15,16]$. These or related traits have similarly been named as risk factors towards emotional eating in obese individuals $[17,18]$. Therefore, psychologically pre-disposed individuals who engaged in eating to cope prior to bariatric surgery could be more likely to use alcohol as a replacement coping mechanism if other self-regulatory measures are not learned or employed.

Beyond drinking to cope, motivations to drink alcohol post-surgery may also shift according to changes in selfesteem and socialisation. Bariatric surgery changes multiple aspects of patients' lives, including psychological health, social ties, sexual lives, body image, eating behaviour and relationship with food [19]. Therefore, changes in alcohol use including alcohol misuse may also be anticipated.
Understanding the motivations driving alcohol misuse after surgery could also inform clinical interventions aiming to reduce these incidents; however, studies using patient voice and insight are rare. Although few studies have employed qualitative approaches to understanding post-surgical alcohol misuse, the extant literature is nonetheless informative. One study identified several contributors to post-surgical substance misuse, including "unresolved psychological problems" and "addiction transference" [20]. Building upon this, Yoder and colleagues [21] developed a theory using interviews from post-bariatric surgery patients with alcohol use disorder (AUD) specifically. They constructed a "filling the void" model, where patients' previous food-related coping strategies and unresolved psychological issues motivated the development of post-surgical AUD. Importantly, a comparison group of patients is lacking in these qualitative studies, which could reveal additional insight into key factors that differentiate participants with problematic alcohol use in the post-surgery period from those without. Therefore, the present study aimed to interview both participants who do and do not have problematic alcohol use or misuse post-surgery to understand which factors influence the development of post-surgical alcohol misuse.

\section{Method}

\section{Procedure}

Ethical approval was obtained from the university research ethics committee. Participants were identified through advertising on a social media platform for bariatric support groups. After confirming interest, an initial telephone screening procedure was used to ensure all participants met the study criteria of (1) being 18 years or older, (2) receiving bariatric surgery at least 18 months prior as alcohol misuse tends to appear around this time-point $[4,5]$, (3) not being pregnant/ breastfeeding and (4) not having disclosed unmet mental health needs. Upon receiving informed consent, the first author (a doctoral student experienced in qualitative methods with an MSc in health psychology) conducted semistructured interviews with participants using an interview schedule to guide the conversation (see Table 1). The openended and exploratory interview questions were developed by researchers with expertise in qualitative research, eating behaviour and substance misuse. Following a literature review, specific question items were included to invite participants to reflect on their relationship with alcohol before and after surgery, with additional questions targeting possible triggers for problematic alcohol use (e.g. unmet expectations, life events) based on previous studies $[5,22,23]$. The in-person interviews took place in a familiar setting, including the participant's home, community location or the university. Interviews 
Table 1 Interview schedule for the present study. Italicised questions are prompts to encourage further discussion
1. Demographic information

Age, relationship status, type of bariatric surgery received and when, current weight, weight loss since surgery

2. How would you describe your relationship to food (or "eating style") before surgery? Has this changed now that you have had bariatric surgery?

How so?

3. Before your bariatric procedure, did you drink alcohol?

If yes - How often per week? How would you describe your pre-surgery relationship with alcohol?

If no - Why not?

4. What were your expectations towards the results of your bariatric surgery?

Lose a specific amount of weight, feel a certain way, changes in areas of your life?

Do you think your results have met those expectations?

5. Did you experience any difficulties adjusting to new habits or routines after your bariatric surgery?

If yes - What were some of those difficulties? Do you feel that you have overcome them? What helped you overcome them?

If no - What made your adjustment go well?

6. Were there any major life events that occurred prior to your surgery, or afterwards, that you felt impacted your recovery and adjustment post-surgery?

If yes - What were they?

7. Have you drank alcohol since you have had weight loss surgery?

If yes - Does it affect you differently now than before the surgery? How would you describe your relationship with alcohol at present?

If no - What are your reasons for not drinking alcohol?

8. Are you happy with the results of your surgery - would you, given the chance, do it all over again, knowing what you know now?

If yes - What factors influenced your answer?

If no - What would you have done differently?

9. If you could give advice to someone considering bariatric surgery, what would you want to say to them? What advice would you have for the clinical care team? were audio recorded, lasted approximately 1 hour, and all participants were offered $£ 20$ (approx. \$26) gift cards as compensation for their time and contribution to the study. Audio interviews were transcribed verbatim, anonymised and imported into the qualitative data analysis software package NVivo10. [24]. After each interview, the participants were debriefed and invited to contact the principal investigator with additional concerns or questions regarding the study.

\section{Participants}

Overall, the number of interviews was guided by data saturation, and 14 participants completed an interview either in person $(n=6)$ or over the telephone for convenience $(n=8$; see Table 2). Problematic alcohol use classifications were assigned using responses to interview questions. If a participant described drinking at hazardous levels, being advised by a medical professional to reduce their drinking, difficulty with controlling their intake, seeking assistance or support to reduce their drinking, and/or expressed concern or guilt that alcohol had a prominent role in their lives and had not made efforts to discontinue or reduce their drinking, then their drinking was classified as "problematic alcohol use" (PAU). At the time of the interview, four of the six participants with PAU had discontinued or modified their drinking habits independently or otherwise sought help through a general practitioner (GP), mental health or community service. Participants without problematic alcohol use were classified as "non-problematic alcohol use" (NPAU). All participants were given a pseudonym, and identifiable details were omitted from the transcript.

\section{Thematic Analysis}

The thematic analysis used in this study was informed by the inductive method described by Braun and Clarke [25]. First, transcripts were read iteratively to generate ideas through data immersion. Second, initial codes were systematically generated within and across the full dataset. The third and fourth phases of analysis involved collecting the codes (and relevant data) into potential themes and reviewing themes for overlapping/dissimilar content, and further refining through separating or grouping themes between transcripts. This process generated a thematic "map" of the analysis and ensured clear thematic distinction. The final themes were checked 
Table 2 Participant characteristics for participants with $(n=6)$ and without problematic alcohol use $(n=8)$. Values are counts (gender, surgery type), means, $95 \%$ confidence intervals $(\mathrm{CI})$ and effect size values for between-group differences

\begin{tabular}{lllll}
\hline & $\begin{array}{l}\text { With problematic } \\
\text { alcohol use }\end{array}$ & $\begin{array}{l}\text { Without problematic } \\
\text { alcohol use }\end{array}$ & $\begin{array}{l}\text { Effect } \\
\text { size }\end{array}$ & $\begin{array}{l}\text { 95\% CI } \\
\text { (UL, LL) }\end{array}$ \\
\hline $\begin{array}{l}\text { Gender (female/male) } \\
\text { Surgery type }\end{array}$ & $4 / 2$ & $5 / 3$ & $0.04^{\mathrm{b}}$ & - \\
$\quad$ Roux-en-Y gastric bypass & 5 & & $0.35^{\mathrm{c}}$ & - \\
Sleeve gastrectomy & - & 5 & - & - \\
Other & 1 & 1 & - & - \\
Age (years) & 51.83 & 2 & - & - \\
Years since surgery & 8.83 & 43.13 & $1.08^{\mathrm{a}}$ & $(2.21,0.05)$ \\
Current weight (kg) & 105.79 & 5.06 & $0.91^{\mathrm{a}}$ & $(2.07,0.20)$ \\
Post-surgery weight loss (kg) & 47.09 & 98.06 & $0.27^{\mathrm{a}}$ & $(1.34,0.79)$ \\
\hline
\end{tabular}

Effect size values were calculated using ${ }^{\mathrm{a}}$ Cohen's $d,{ }^{\mathrm{b}} \mathrm{Phi}(\Phi)$ for nominal data and ${ }^{\mathrm{c}}$ Cramer's $V\left(\varphi_{c}\right)$ for nominal data with multiple categories

There was no effect $(\Phi=0.04)$ for gender between participants with PAU and NPAU. However, there was a medium effect $\left(\varphi_{c}=.35\right)$ for surgery type, large $(d=1.08)$ for age, large $(d=0.91)$ for years since surgery, small $(d=0.27)$ for current weight and medium $(d=0.49)$ for post-surgery weight loss. Therefore, all factors except gender held meaningful between-group differences for participants in this study, with participants with NPAU having a surgery type other than Roux-en-Y gastric bypass, being younger on average, having fewer years since surgery, lower current weight and greater post-surgery weight loss. However, due to small sample sizes any differences should be treated with caution against the coded extracts and the full dataset. Once key themes had been identified, the final stage included defining which data qualities each theme captured, and a detailed analysis was written to describe the theme, including relevant sub-themes. To verify validity and reliability, a second author coded a subset of extracted data representative of key codes and themes from 10 transcripts and compared the overall agreement. The target level of $80 \%$ agreement was reached $(K=0.80)$ and discrepancies were resolved on a case-by-case basis until reaching full agreement $(K=1.00)$. A third author with expertise in qualitative methods reviewed the final thematic map and analysis.

\section{Results}

The participants provided insightful, descriptive accounts of their experiences before and after bariatric surgery. Five core themes were identified in both the participants with PAU and with NPAU, with a sixth core theme ("resilience") identified specifically in participants with NPAU. Sub-themes within each major theme are further detailed and depicted in Fig. 1.

\section{Theme 1: Drinking Motivations}

Drinking was often influenced by external factors such as negative life events or social occasions, with key differences appearing in motivations and behaviours between PAU and NPAU groups. While participants with PAU expressed drinking alcohol to cope with negative affect, surgical restriction, transferring their coping habits onto alcohol from food, and feelings of disinhibition, participants with NPAU endorsed social motivations or described strategies for maintaining control over drinking.

\section{1a Problematic Alcohol Use: Coping and Disinhibition}

Overall, drinking to cope was a core motivation driving problematic alcohol use post-surgery, which had either been a habit continued from pre-surgery (two of the six participants with PAU) or had newly onset following surgery. For participants with PAU, drinking alcohol functioned as a coping mechanism to regulate negative affect, "... the worst times [were] when I was using it as a coping strategy" [Sandy, PAU]. In other narratives, this appeared to be a "transfer" from previously food-oriented coping strategies before surgery to alcohol. One participant offered "it's either there because I need a reward or ... because I need some comfort. I think maybe the feelings that I had with food goes into that glass of wine" [Jane, PAU]. Disinhibition over drinking was another motivation, with several participants describing feeling intoxicated quickly once they began drinking, which led to feeling less able to control their intake or set limits for themselves after their drinking had started, which led to drinking more than anticipated, "once I start drinking, if I have one or two drinks I lose the ability to not have any more" [Sandy, PAU].

\section{1b. Non-problematic Alcohol Use: Social and Maintaining Control}

Social drinking was endorsed by all but one participant, and maintaining control over alcohol use appeared specifically within participants with NPAU. For participants who were socially 


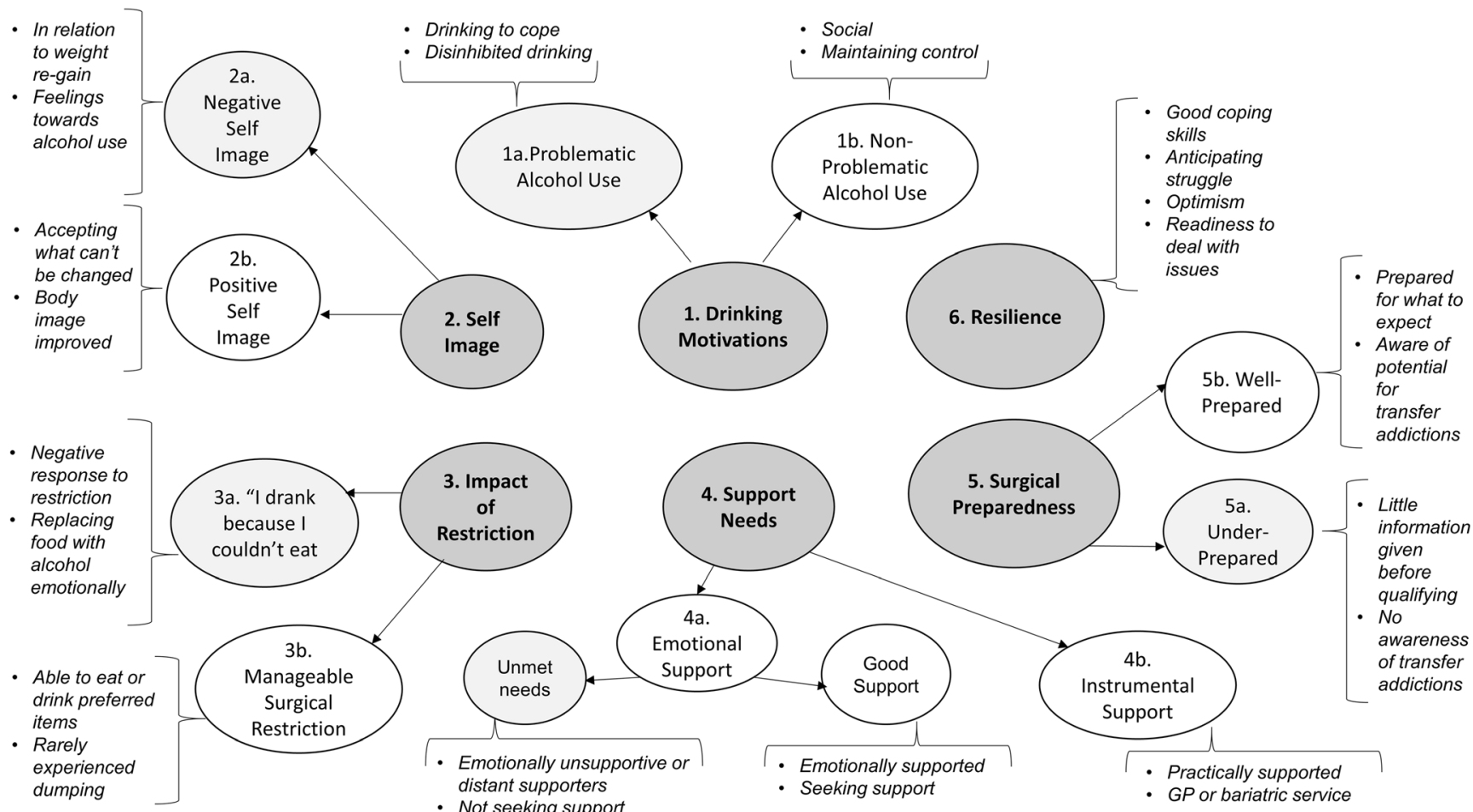

Fig. 1 Themes and sub-themes assigned for participants with problematic alcohol use [PAU] and with non-problematic alcohol use [NPAU]. Themes are numbered and in bold, sub-themes are numbered and in non-italics, and codes are bullet-pointed and italicised. Sub-themes belonging to PAU are in light greyscale, and those for NPAU have no colour

\section{2a. Negative Self-image}

motivated like Jennifer [NPAU], spending time with people was the key motivator, “...the focus wasn't on the drinking, it was on the meeting people and talking." Also, many participants also felt as though drinking was not required in every social circumstance, and occasionally abstained from alcohol. Maintaining control was evident where four participants recognised the possibility of falling into a pattern of using alcohol to cope and explained their strategies for reducing their drinking when they felt vulnerable, including avoiding alcohol altogether. Bridget [NPAU] gave an example of avoiding alcohol when she felt upset, "I was like '... what if alcohol becomes a problem' because I used to use food to control my emotions... and actually I haven't drunk since." Altogether, some participants with NPAU indicated awareness that drinking to cope was possible and kept their drinking mostly socially occasioned.

\section{Theme 2: Self-image}

The second theme associated with problematic alcohol use is drawn from the thoughts and feelings participants had about their outward appearances, internal dialogues and beliefs, conceptualised as a negative or a positive self-image. Participants with PAU endorsed more negative self-images, which stemmed from their alcohol use and body image. Participants with NPAU, on the other hand, endorsed more positive self-images, improvements in body image and selfacceptance.
For some participants with PAU, a negative self-image appeared connected to feeling disappointed about their problematic relationship with alcohol after surgery, and its consequences upon weight re-gain. Weight re-gain was a source of shame or frustration for half of the participants with PAU, and often led to negative feelings and self-consciousness. When looking at a photograph, Walter [PAU] described, “... we're all post-surgery, but I felt I was the worst... thinking about it, I probably wasn't. Maybe I need to adjust that.” A couple of the transcripts revealed that not only did this negative self-image affect them personally but also contributed to a less forgiving attitude towards others who have re-gained weight after surgery, although this was similarly observed in three of the interviews from participants with non-problem alcohol use. Nonetheless, there were instances of participants with PAU speaking positively about their surgical results or re-framing weight re-gain as acceptable. Taken together, negative self-image occurred in PAU narratives regarding drinking behaviours and related consequences, but also seemed continued from experiencing weight stigma before surgery.

\section{2b. Positive Self-image}

Participants without problematic alcohol use drew their positive self-images from receiving encouragement from other 
patients, and positive feelings towards or acceptance of surgical results. Patrick [NPAU] articulated, “... the dietician would say it hasn't been a total success because I haven't lost $100 \%$ of my excess weight.... I would think the surgeons would be quite happy because I've lost over $25 \%$ of my body weight.. and therefore, from their point of view, it's undoubtedly a success. And I'm very much in that camp...." 'Further, being treated like "a normal person" was cited by a couple of the participants with NPAU, which contributed to self-image improvements. This desire to be treated "normally" was expressed by all participants and may have stemmed from internalising stigmatisation that many persons with obesity endure before surgery. Despite a few instances where participants with NPAU could be self-critical, generally they appeared optimistic that they could make positive changes through their own effort, or had access to help from outside sources, such as plastic surgery.

\section{Theme 3: Impact of Restriction on Eating Behaviour}

Another contributor to problematic alcohol use was the impact of the surgically imposed restriction on eating behaviour. All but one of the participants with PAU cited the inability to eat as underpinning drinking alcohol problematically, while most of the participants with NPAU reflected that their surgical restriction was more manageable, including being able to eat "bad" foods and struggling to limit their emotional eating.

\section{3a. "I drank because I couldn't eat"}

Most participants with PAU described the impact restriction had upon their ability to eat foods that they would have previously used to comfort themselves, or commonly eaten foods (e.g. rice). Alcohol, on the other hand, did not pose the same pitfalls that over-indulging in these foods did, "... I could eat a sweetie bar and be crippled over with pain [laughs], but I could drink a bottle of wine and be absolutely fine" [Sandy, PAU]. In social eating circumstances, having small portions of less desired foods detracted from the pleasurable quality of the experience. Drinking, however, offered a solution to engage and find satisfaction, "filling the gap" that having fewer food options left in their lives. "... drinking became something you could do because it wasn't eating.... I had a relationship with food that wasn't simple, and it was changed, and I wanted something to fill it" [Walter, PAU]. Not being able to eat as much, or the same foods, could foster a negative emotional response to the restriction that surgery imposed on participants with PAU.

\section{3b. Manageable Surgical Restriction}

Although participants with NPAU also experienced surgical restriction and "dumping" (a reaction to foods high in sugar/ carbohydrates comprised of nausea, sweating, fatigue and diarrhoea symptoms), nearly all described it as manageable. Two participants described finding ways around the restriction, including choosing specific foods, less likely to cause dumping-related symptoms. Ben [NPAU] found himself still able to emotionally eat after surgery, which was something he made efforts to discontinue "... and [I] stopped it, and I got back to my lowest weight. Definitely, looking back, I was on the track to go back to where I was." Feeling deprived of the positive emotional experiences associated with food was a major difference between participants with PAU and NPAU. Participants with NPAU largely remained able to enjoy rewarding foods, even if they had to choose wisely or alter the amounts they could eat.

\section{Theme 4: Support Needs}

Both participants with PAU and NPAU described having sources of support, including a partner, family members, communities or medical teams. Whether those structures offered the support that participants needed appeared to be a contributing factor towards problematic alcohol use. Participants with PAU tended to have unmet emotional support needs, while many participants with NPAU described seeking sources of emotional support and receiving support as being essential throughout the surgical pathway.

\section{4a. Emotional Support}

All participants with PAU had supportive people in their lives, but the level of emotional support they received when their relationship to alcohol was problematic appeared inconsistent, insufficient or absent. Although many described their supporters as instrumentally helpful with food preparation or surgery recovery, emotional support needs continued - at times because they did not seek support when they were having difficulty coping "... I felt awful keeping it from [family member]" [Martha, PAU]. In other instances, participants with PAU felt unable to share their emotional experience with their supporters, which may have left them with unmet needs to be filled by alcohol. A few participants with NPAU described their supporters cheering them on or keeping their spirits up during difficult times; "I think you're really lucky to find the right person... even when things are really tough, sitting there and having a giggle over things, because it's the way you get through." [Jennifer, NPAU]. Relationships also changed for some participants after surgery, possibly to find more emotionally supportive partners. Seeking emotional support throughout the surgical experience was prominent within the narratives, and often participants with PAU began to address their relationship with alcohol after seeking emotional support. 


\section{4b. Instrumental Support}

Both participants with PAU and NPAU alike had mixed experiences with instrumental support from their medical teams, including bariatric surgeons, dieticians and GPs. Many participants in both groups disliked the time-limited nature of bariatric pathway support provision, "Once those two years are up that's it, you know, they kind of cut the ties...." [Martha, PAU]. Other participants had a positive experience, and some acknowledged that it felt like a lottery that they happened to be on the winning side of; "[Surgeon]... his team is absolutely amazing, you can call them up for advice.... I think should be mandatory for every place." [Kristen, NPAU]. While there was a distinctive difference between the levels of emotional support participants received after surgery, instrumental support varied within both groups. Instrumental support was also received from the closest people, and areas for assistance included food preparation and surgery recovery.

\section{Theme 5: Surgical Preparedness}

Preparedness for the realities following bariatric surgery involved having sufficient information to help transition into a different lifestyle to accommodate restriction and being prepared for weight loss. Feeling underprepared was a possible contributor towards problematic alcohol use, as many described not receiving enough information at their presurgical appointments or having any awareness of the possibility for "addiction transference" to alcohol. Five in the NPAU group, however, felt they had prepared well for surgery through support group attendance, personal research or having a good experience in their bariatric service.

\section{5a. Underprepared}

Four individuals in the PAU group described not having enough information prior to bariatric surgery, leaving doubts regarding what to eat, what side effects to be wary of, and potential psychological adjustments “... but I did feel completely lost at each stage and I didn't feel I really knew volumes" [Jane, PAU]. A key component of feeling underprepared for surgery included not knowing that alcohol could become a problem. Half of the participants with problem alcohol use felt as though more information would have helped shape their expectations for how their relationship with alcohol would change "... the surgeon didn't say to me once 'oh it could drastically affect the way your body absorbs alcohol'... It says in the leaflets... you can drink in moderation. Well no, I can't drink in moderation" [Sandy, PAU].

\section{5b. Well Prepared}

For participants with NPAU, preparation came from multiple sources, including doing their own personal research, attending a support group or having an informative bariatric pathway experience. Further to being aware of the changes and challenges following surgery, a few participants felt as though their personal research and inquiries informed the effort they later made to avoid over-consuming alcohol; "... I did something like 2 years of research before I actually got my surgery.... I was lucky because it was something that I was on the lookout for, rather than people who go into it blind" [Jennifer, NPAU]. Going into surgery "blind," or underprepared was an experience that most participants with NPAU sought to avoid, and their efforts distinguished the two groups in terms of how prepared they felt they were for bariatric surgery and the potential for problematic alcohol use.

\section{Theme 6: Resilience}

The sixth core theme was "resilience," characterised by the presence of self-confidence, readiness to address mental health, optimism and good coping skills, which increased the capacity for participants with NPAU to cope with difficulties, both surgery-related and in their personal lives. For some participants like Karen [NPAU], investing time in a mental health service helped to identify previous coping habits, “... they helped me sort of realise... it's ok if everything is not perfect all the time. Because that was a big thing - if something wasn't right, I would turn to food." Replacing the previous coping strategy of emotional eating with more effective, accessible coping skills was a key feature of resilient participants with NPAU. Throughout the transcripts, other skills included setting manageable goals, acceptance, identifying triggers, avoidance and connecting with others. For the participants with PAU, those that had later managed or resolved their problematic alcohol use through personal effort, a mental health service or a GP intervention described a mindset shift contributing towards changing their relationship to alcohol. After getting help, Martha [PAU] described feeling more empathetic towards others, and challenging the guilt she internalised for re-gaining weight, "Who am I to judge? And it's just seeing first-hand what drugs and alcohol can do to people that I think 'hang on a minute, there's a lot more to life that matters...." In summary, participants with NPAU maintained resilience despite the adversities that follow the major life changes inherent to and outside of surgery. Also, cultivating a more resilient mindset may have helped some participants with PAU navigate away from coping maladaptively with alcohol. 


\section{Discussion}

The current study aimed to understand what factors influence the development of alcohol misuse after bariatric surgery by interviewing participants with differing relationships to alcohol, and several informative themes emerged. Overall, participants with PAU cited drinking to cope and disinhibition as influential to developing problematic alcohol use. Primarily, findings support the theory developed by Yoder and colleagues [21], where previously eating-centred coping mechanisms for unresolved psychological problems before surgery and experiencing a "new buzz" from the rapid effects of alcohol post-surgery contributed to drinking to cope. Conversely, participants with NPAU reduced their drinking when they became concerned, and mostly kept their drinking socially occasioned. In this way, including a comparison group revealed that some individuals were aware of this potential to misuse alcohol, and employed strategies to manage the new effects of alcohol. Together, these themes emphasise the role that coping behaviours and awareness play in the development of post-surgical problematic alcohol use.

It has been suggested that post-surgery new-onset substance misuse reflects "addiction transference" from food to other substances [26, 27]. There is evidence that common neural systems drive the rewarding effects of food and substances such as drugs and alcohol [28]. Furthermore, RYGB has been shown to reduce brain reward system activation to high-calorie foods (relative to the gastric band) [29]. However, a recent systematic review [30] reported mixed evidence for the relationship between pre-surgical food addiction and postsurgical SUD/AUD. Themes identified in the present study go beyond the "addiction transference" model and provide insight into the negative reinforcement mechanisms (i.e. drinking to cope) driving post-surgical alcohol misuse. This is consistent with evidence from Yoder and colleagues [21] and supports the theory that switching from eating to alcohol use post-surgery represents seeking a replacement behaviour to cope with underlying vulnerabilities [3].

Results also illustrate that the emotional cost of disappointment with weight or surgical outcomes could open an avenue for patients to consume alcohol as a strategy to cope. Despite instances of positive perspectives, a comparatively negative self-image was described amongst participants with PAU. This was especially evident regarding weight re-gain and body image and extends the literature where increases in self-esteem correspond to reductions in BMI post-surgery [31]. Another surgical outcome influencing drinking to cope was surgery's impact upon eating behaviour, where all but one of the participants with PAU "drank because [they] could not eat." At times, this appeared connected to dumping syndrome (it is estimated that up to $40 \%$ of patients may experience dumping after surgery) [32], and some with PAU also described alcohol as emotionally comforting. In both contexts, drinking alcohol "fills the gap" created by losing the ability to eat these foods, whereas participants with NPAU mostly described their restriction as manageable. This provides new evidence for the potential to "switch" coping mechanisms from food to alcohol for some whose ability to eat or relationship to food changes post-surgery [3, 21].

In bariatric surgery literature, the role of social support and alcohol use is under-examined, as outcomes mainly target weight loss. Often, social support is linked to positive outcomes, with a wider network contributing to greater weight loss [33]. Studies in non-bariatric populations link receiving less social and emotional support to an increased likelihood to drink heavily [34]. The present study indicates that unmet emotional support needs could similarly influence the development of problematic alcohol use post-bariatric surgery, possibly by maladaptively coping with the lifestyle, relational and psychological changes that arise. Similarly, high surgical expectations may be related to psychological distress if the expectations are unmet, and Kubik et al. [22] emphasised that the pre-surgical evaluation is an opportunity to identify patients needing more support and information. Narratives from participants with NPAU in the current study provide further insight on surgical preparedness, as many described conducting their own research, speaking with other patients and feeling informed from the bariatric pathway. Additional research would further illuminate the role of both support systems and surgical preparedness in the development of problematic alcohol use following surgery.

While providing external support is critical, fostering internal "resilience" resources in bariatric patients is another purpose of professionals and support groups [35]. Findings provide evidence for the role of resilience by identifying that participants with NPAU possessed self-confidence, readiness to address mental health issues, optimism and good coping skills. Together, these qualities may promote recovery from surgery-related and personal difficulties, thereby preventing the development (and maintenance) of drinking to cope. Correspondingly, research comparing post-bariatric surgery patients found that individuals who developed substance use disorders (SUDs) reported more stressful life events following surgery and coping through substance use [23]. Results from the present study reflect a similar tendency, as drinking to cope motivated alcohol misuse or feeling concerned over drinking habits. Longer-term follow-up is merited to investigate how resilience develops and changes post-surgery, as participants with PAU were interviewed at a comparatively longer time following surgery relative to participants with NPAU.

Clinical applications of the present study's findings could inform elements of a personalised pre- and post-surgical intervention strategy, for example, informing patients about the increased risk for alcohol misuse post-surgery, changes in alcohol's physiological effects, considering patients' existing coping strategies and facilitating tailored psychological 
support during the post-surgical period [3]. Responses from the interviews highlighted a need for pre-surgical counselling, follow-up and service accessibility after surgery. Multidisciplinary teams could promote preparedness by offering pre-surgery counselling regarding lifestyle changes due to gastric restriction. Further, post-surgical support for patients experiencing feelings of deprivation around food could be helpful to mitigate the impact of surgical restriction and help to develop positive coping strategies. Based on the present study's findings, interventions within research and clinical settings could also investigate increasing patient resilience through addressing self-image, mental health and educating patients about available coping skills and strategies.

\section{Limitations}

A potential limitation is that six interviews were conducted in person (participants with PAU $n=4$; NPAU $n=2$ ), and eight were conducted over the telephone (PAU $n=2$; NPAU $n=6$ ). While this strategy increased participation from participants who might have been restricted geographically or otherwise, there is potential for disadvantages including the lack of visual cues or environmental distractions [36, 37]. Telephone interviewing is described as a flexible data collection method of comparable quality with in-person interviews [38]. Future research comparing methodology in populations with problematic alcohol use is merited, especially regarding sensitive information disclosure. Another limitation is the largely retrospective nature of accounts from participants, and a longitudinal design could better capture the developmental aspect of problematic alcohol use. Regardless, the findings insightfully draw upon the participants' reflective experiences of problematic and non-problematic alcohol use post-surgery. Participant responses were also used to assign participants to their problematic alcohol use group (with vs. without), as interview questions elicited responses about post-surgical alcohol use that external validation measures (e.g. questionnaire-based assessment of current alcohol use) might not have captured for participants who had already discontinued or modified their drinking habits at the time of the interview. However, despite its advantages, this classification method has limitations in terms of validity, and future research could address this limitation through identifying and applying external validation measures assessing the history of alcohol problems post-surgery. Moreover, potential group differences are illustrated in Table 2 where participants with NPAU appear more likely to have a surgery type other than RYGB, be younger, have fewer years since surgery, lower current weight and greater postsurgery weight loss compared with participants with PAU. However, due to small sample sizes, any differences should be treated with caution.

\section{Conclusions}

Results from the current study identify several themes implicated in the development of problematic alcohol use after bariatric surgery. Participants with problematic alcohol use endorsed drinking to cope and disinhibited drinking motivations, a more negatively perceived self-image, adverse impacts of surgical restriction, receiving less emotional support or having unmet needs and feeling underprepared for surgery in terms of expectations regarding alcohol use. Conversely, narratives from participants with non-problematic alcohol use were marked by social motivation or maintaining control over drinking, a more positive self-image, manageable surgical restriction, having sufficient emotional support, feeling more prepared for surgery and more resilience contributing to the capacity to endure difficulties following surgery.

Acknowledgements We affirm that all personally identifying information has been removed or altered so the participants cannot be identified through included quotes or details.

Funding Information All funding was provided by the University of Liverpool Institute of Psychology, Health and Society.

\section{Compliance with Ethical Standards}

Conflict of Interest The authors of this manuscript declare no relevant competing interests. Paul Christiansen, Jason C. G. Halford and Charlotte A. Hardman receive funding from the American Beverage Association. Charlotte A. Hardman has also received speaker fees from the International Sweeteners Association.

Statement of Informed Consent Informed consent was obtained from all individual participants included in the study.

Statement of Human and Animal Rights/Ethical Approval Ethical approval was obtained from the university research ethics committee (REF 0422).

Open Access This article is distributed under the terms of the Creative Commons Attribution 4.0 International License (http:// creativecommons.org/licenses/by/4.0/), which permits unrestricted use, distribution, and reproduction in any medium, provided you give appropriate credit to the original author(s) and the source, provide a link to the Creative Commons license, and indicate if changes were made.

\section{References}

1. Picot J, Jones J, Colquitt J, et al. The clinical effectiveness and costeffectiveness of bariatric (weight loss) surgery for obesity: a systematic review and economic evaluation. Health Technol Assess. 2009;13(41):1-190. 215-357, iii-iv

2. Buchwald $\mathrm{H}$, Avidor $\mathrm{Y}$, Braunwald E, et al. Bariatric surgery: a systematic review and meta-analysis. JAMA. 2004;292(14):1724-37.

3. Hardman C, Christiansen P. Psychological issues and alcohol misuse following bariatric surgery. Nat Rev Endocrinol. 2018;14(7): $377-8$. 
4. Conason A, Teixeira J, Hsu C, et al. Substance use following bariatric weight loss surgery. JAMA Surg. 2013;148(2):145-50.

5. King W, Chen J, Mitchell J, et al. Prevalence of alcohol use disorders before and after bariatric surgery. JAMA. 2012;307(23):2516-25.

6. Svensson P, Anveden Å, Romeo S, et al. Alcohol consumption and alcohol problems after bariatric surgery in the Swedish obese subjects study. Obesity. 2013;21(12):2444-51.

7. Hagedorn J, Encarnacion B, Brat G, et al. Does gastric bypass alter alcohol metabolism? Surg Obes Relat Dis. 2007;3(5):543-8.

8. Mechanick J, Youdim A, Jones D, et al. Clinical practice guidelines for the perioperative nutritional, metabolic, and nonsurgical support of the bariatric surgery patient-2013 update: cosponsored by American Association of Clinical Endocrinologists, The Obesity Society, and American Society for Metabolic \& Bariatric Surgery. Endocr Pract. 2013;19(2):337-72.

9. Spadola C, Wagner E, Dillon F, et al. Alcohol and drug use among postoperative bariatric patients: a systematic review of the emerging research and its implications. Alcohol Clin Exp Res. 2015;39(9): 1582-601.

10. Lent M, Hayes S, Wood G, et al. Smoking and alcohol use in gastric bypass patients. Eat Behav. 2013;14(4):460-3.

11. Wee C, Mukamal K, Huskey K, et al. High-risk alcohol use after weight loss surgery. Surg Obes Relat Dis. 2014;10(3):508-13.

12. Burgess E, Turan B, Lokken K, et al. Profiling motives behind hedonic eating. Preliminary validation of the Palatable Eating Motives Scale. Appetite. 2014;72:66-72.

13. Boggiano M, Wenger L, Turan B, et al. Eating tasty food to cope. Longitudinal association with BMI. Appetite. 2015;87:365-70.

14. Merrill J, Thomas S. Interactions between adaptive coping and drinking to cope in predicting naturalistic drinking and drinking following a lab-based psychosocial stressor. Addict Behav. 2013;38(3):1672-8.

15. Baines L, Jones A, Christiansen P. Hopelessness and alcohol use: the mediating role of drinking motives and outcome expectancies. Addict Behav Rep. 2016;4:65-9.

16. Holahan C, Moos R, Holahan C, et al. Drinking to cope and alcohol use and abuse in unipolar depression: a 10-year model. J Abnorm Psychol. 2003;112(1):159-65.

17. Elfhag K, Morey L. Personality traits and eating behaviour in the obese: poor self-control in emotional and external eating but personality assets in restrained eating. Eat Behav. 2008;9(3):285-93.

18. Schneider K, Appelhans B, Whited M, et al. Trait anxiety, but not trait anger, predisposes obese individuals to emotional eating. Appetite. 2010;55(3):701-6.

19. Coulman K, MacKichan F, Blazeby J, et al. Patient experiences of outcomes of bariatric surgery: a systematic review and qualitative synthesis. Obes Rev. 2017;18(5):547-59.

20. Ivezaj V, Saules K, Wiedemann A. "I Didn't See This Coming.": Why are postbariatric patients in substance abuse treatment? Patients' perceptions of etiology and future recommendations. Obes Surg. 2012;22(8):1308-14.
21. Yoder R, MacNeela P, Conway R, et al. How do individuals develop alcohol use disorder after bariatric surgery? A grounded theory exploration. Obes Surg. 2017;28(3):717-24.

22. Kubik J, Gill R, Laffin M, et al. The impact of bariatric surgery on psychological health. J Obes. 2013;2013:1-5.

23. Ivezaj V, Saules K, Schuh L. New-onset substance use disorder after gastric bypass surgery: rates and associated characteristics. Obes Surg. 2014;24(11):1975-80.

24. QSR International [Internet]. Qsrinternational.com. 2012 [cited 2018 Aug 22]. Available from: http://qsrinternational.com/

25. Braun V, Clarke V. Using thematic analysis in psychology. Qual Res Psychol. 2006;3(2):77-101.

26. Spencer, J. The new science of addiction: alcoholism in people who had weight loss surgery offers clues to roots of dependency [Internet]. Wall Str. J. 2006 [cited 2018 Oct 23]. Available from https://www.wsj.com/articles/SB115318049135509251

27. Steffen KJ, Engel SG, Wonderlich JA, et al. Alcohol and other addictive disorders following bariatric surgery: prevalence, risk factors and possible etiologies. Eur Eat Disord Rev. 2015;23(6):442-50.

28. Volkow ND, Wang GJ, Tomasi D, et al. Obesity and addiction: neurobiological overlaps. Obes Rev. 2013;14(1):2-18.

29. Scholtz S, Miras AD, Chhina N, et al. Obese patients after gastric bypass Surgery have lower brain-hedonic responses to food than after gastric banding. Gut. 2014;63(6):891-902.

30. Ivezaj V, Wiedemann AA, Grilo CM. Food addiction and bariatric surgery: a systematic review of the literature. Obes Rev. 2017;18(12):1386-97.

31. Burgmer R, Legenbauer T, Müller A, et al. Psychological outcome 4 years after restrictive bariatric surgery. Obes Surg. 2014;24(10): $1670-8$.

32. van Beek A, Emous M, Laville M, et al. Dumping syndrome after esophageal, gastric or bariatric surgery: pathophysiology, diagnosis, and management. Obes Rev. 2016;18(1):68-85.

33. Livhits M, Mercado C, Yermilov I, et al. Is social support associated with greater weight loss after bariatric surgery?: a systematic review. Obes Rev. 2011;12(2):142-8.

34. Strine T, Chapman D, Balluz L, et al. Health-related quality of life and health behaviours by social and emotional support. Soc Psychiatry Psychiatr Epidemiol. 2007;43(2):151-9.

35. Sarvey S. Psychosocial support after bariatric surgery: fostering resilience. Bariatric Nurs Surg Patient Care. 2009;4(4):323-4.

36. Garbett R, Mccormack B. The experience of practice development: an exploratory telephone interview study. J Clin Nurs. 2001;10(1): 94-102.

37. Opdenakker R. Advantages and disadvantages of four interview techniques in qualitative research. Forum Qual Soc Res. 2006;7(4)

38. Carr E, Worth A. The use of the telephone interview for research. NT Res. 2001;6(1):511-24.

Publisher's Note Springer Nature remains neutral with regard to jurisdictional claims in published maps and institutional affiliations. 